\title{
Análise de 29 casos de esclerite. Experiência de um serviço de Reumato-Oftalmologia
}

\author{
Analysis of 29 cases of scleritis. Experience of a Rheumato-Ophthalmology \\ Service
}

Andreo Garcia Morante Parra ${ }^{1}$

Fernando Heidi Miyazaki ${ }^{2}$

Ramiro Magalhães Ribeiro ${ }^{3}$

Marcelo Luis Gehlen ${ }^{4}$

Thelma Skare ${ }^{5}$

\begin{tabular}{|l|}
\hline RESUMO \\
\hline Esclerites são doenças que podem ter como causa uma doença reuma- \\
tológica, infecciosa ou tumoral ou, ainda, ser uma entidade restrita \\
apenas ao olho. Objetivo: Relatar a experiência de dois anos de um \\
ambulatório conjunto de Reumato-Oftalmologia no diagnóstico e acom- \\
panhamento de pacientes com esclerite por três anos. Métodos: Esta \\
é uma análise de 29 casos de esclerite. Todos os casos foram avaliados \\
pelo serviço de Oftalmologia e pelo de Reumatologia. Resultados: Dos 29 \\
casos identificados, foi possível identificar uma doença subjacente \\
em 55,17\% dos casos. Observamos doenças reumáticas em 34,4\% e \\
infecciosas 20,6\% dos casos. As esclerites idiopáticas não se distin- \\
guiram das reumáticas quanto às características clínicas embora um \\
maior número de casos com a forma necrosante tenha sido visto nas \\
reumáticas (sem significância estatística). Esclerites com doença reu- \\
mática subjacente foram tratadas mais vezes com corticóides orais e \\
imunossupressores. Conclusões: A cooperação entre reumatologistas \\
e oftalmologistas se mostrou útil na identificação etiológica de pa- \\
cientes com esclerite.
\end{tabular}

Descritores: Esclerite/etiologia; Esclerite/quimioterapia; Artrite reumatóide/quimioterapia; Granulomatose de Wegener/quimioterapia; Imunossupressores/uso terapêutico
Trabalho realizado no Hospital Universitário Evangélico de Curitiba.

Médico Residente do Serviço de Oftalmologia do Hospital Universitário Evangélico de Curitiba - HUEC - $\mathrm{Cu}$ ritiba (PR) - Brasil.

${ }^{2}$ Médico Residente do Serviço de Oftalmologia do HUEC Curitiba (PR) - Brasil.

${ }^{3}$ Médico Residente do Serviço de Oftalmologia do HUEC Curitiba (PR) - Brasil

${ }^{4}$ Médico Preceptor do Serviço de Reumato-Oftalmologia do HUEC - Curitiba (PR) - Brasil.

${ }^{5}$ Médica Preceptora do Serviço de Reumato-Oftalmologia do HUEC - Curitiba (PR) - Brasil.

Endereço para correspondência: Thelma L. Skare. Rua João Alencar Guimarães, 796 - Curitiba (PR)

CEP 80310-420

E-mail: tskare@onda.com.br

Conflito de Interesses: nenhum

Fonte de financiamento: nenhuma

Recebido para publicação em 18.12.2009

Última versão recebida em 10.05.2010

Aprovação em 13.05.2010

\section{INTRODUÇÃ̃O}

Esclerite é o termo usado para descrever inflamação da esclera: túnica branca que envolve o olho e que serve como seu esqueleto ${ }^{(1)}$. Mais frequentemente, se apresenta como forma de inflamação da esclera anterior podendo ser difusa, nodular ou necrosante, mas também, pode afetar a esclera posterior. A grande maioria dos casos é leve e idiopática. Por vezes, podem ocorrer apresentações mais agressivas aumentando o risco de associação sistêmica e de consequências graves para a função do olho provocando perda importante da visão(1).

A esclerite é causada na maioria das vezes por um processo imunologicamente mediado sendo alguns deles associados a doenças sistêmicas e outros restritos unicamente ao olho, numa forma de doença imune órgãoespecífica. Todavia, também pode estar associada a infecções (ex: herpes zoster, herpes simples, pseudomonas, amebíase, tuberculose, sífilis, etc.), uso de bisfosfonados e traumas ${ }^{(1-3)}$.

Quaisquer das vasculites autoimunes podem se associar ao aparecimento de esclerite, porém a artrite reumatóide é descrita como sendo a mais comum ${ }^{(4)}$. Entretanto, o aparecimento de novas armas terapêuticas 
para o tratamento dessa entidade, como os imunobiológicos, assim como a conscientização dos reumatologistas da necessidade de manter o processo inflamatório sob rigoroso controle, vêm mudando esse aspecto epidemiológico. Outras vasculites sistêmicas associadas à esclerite são a granulomatose de Wegener, policondrite recidivante, lúpus eritematoso sistêmico, poliarterite nodosa e arterite de Takayasu ${ }^{(5)}$.

A grande possibilidade de associação de doenças oculares como a esclerite com as doenças reumáticas levou o corpo clínico de nosso hospital a criar um ambulatório de ReumatoOftalmologia. Nele os profissionais destas duas especialidades avaliam o paciente no sentido de procurar esclarecer adequadamente a etiologia do processo assim como manejar de maneira mais segura o uso de imunossupressores.

Dados brasileiros acerca da prevalência das doenças sistêmicas associadas à esclerite são escassos, o que motiva a presente revisão, que representa a experiência de três anos de um ambulatório de Reumato-Oftalmologia.

\section{MÉTODOS}

Foram analisados os prontuários de 29 pacientes com esclerite, diagnosticados no ambulatório de Oftalmologia do Hospital Universitário Evangélico de Curitiba durante os últimos três anos. A avaliação oftalmológica consistiu de medida de acuidade visual, biomicroscopia, tonometria de aplanação e oftalmoscopia indireta. Nos casos suspeitos de esclerite posterior, essa foi afastada por ecografia ocular. De acordo com o protocolo local, após o diagnóstico da entidade oftalmológica, todos esses pacientes foram submetidos à avaliação reumatológica e realização dos seguintes testes laboratoriais: fator reumatóide (FR), fator antinuclear (FAN), VHS, Anca C e Anca P, hemograma, parcial de urina, PPD, RX de tórax, além de outros testes que pudessem vir a ser necessários de acordo com a história e exame físico obtidos.

Os dados coletados foram os demográficos, do tipo de esclerite e acuidade visual, diagnóstico final e tratamento utilizado. Esses foram colocados em tabelas de frequência e contingência sendo utilizados os testes de Fisher e Qui-quadrado e Mann Whitney conforme a variável analisada com auxilio do Software Graph Pad Prism, versão 4.0. A significância adotada foi de $5 \%$.

\section{RESULTADOS}

Os dados referentes às características dos pacientes, causas identificadas e tratamento estão na tabela 1 .

Comparando-se as características das esclerites idiopáticas com as de fundo reumatológico observaram-se os dados da tabela 2 .

No que se refere a uni ou bilateralidade do processo inflamatório notou-se que $40 \%$ das esclerites associadas a doenças reumáticas eram bilaterais, contra 22,2\% das idiopáticas.
Todos os casos associados com infecções eram unilaterais. Comparando-se a prevalência de esclerite bilateral nas doenças reumáticas com as idiopáticas obteve-se p=0,62.

\section{DISCUSSÃO}

O diagnóstico etiológico de um quadro de esclerite é quase sempre um grande desafio para o médico atendente. Entretanto o esclarecimento deste aspecto é de suma importância não só porque a etiologia do processo pode guiar a terapêutica adequada como pode levar à descoberta de doenças sistêmicas cuja manifestação inicial é a ocular.

A análise dos 29 casos observados em três anos mostra um porcentual de diagnóstico da doença subjacente em torno de $55 \%$ dos casos, o que evidencia a grande vantagem do acompanhamento do paciente por dois especialistas: o oftalmologista e o reumatologista. A análise de uma coorte americana ${ }^{(5)}$ com 134 pacientes diagnosticados durante 12 anos mostrou um porcentual de identificação de doença subjacente de $47 \%$. Neste estudo $8 \%$ tinham doença infecciosa e $39 \%$ tinham doença reumática, sendo este último valor próximo do encontrado na presente análise. A prevalência dessas entidades varia de acordo com a área geográfica estudada, dadas as influências do perfil genético da população na etiologia das doenças de origem reumática ${ }^{(6)}$ e das condições sociais e cli-

\begin{tabular}{|lc|}
\hline \multicolumn{2}{|c|}{ Tabela 1. Características de 29 casos de esclerites } \\
Dados demográficos \\
Idade \\
Máxima & 72 anos \\
Mínima & 16 anos \\
Média & $59,66 \pm 12,73$ anos \\
Sexo & \\
Masculino & $11-37,93 \%$ \\
Feminino & $18-62,07 \%$ \\
Aspectos oculares & \\
Nodular & $13 / 29-44,82 \%$ \\
Difusa & $11 / 29-37,93 \%$ \\
Necrosante & $5 / 30-17,24 \%$ \\
Posterior & 0 \\
Etiologia & $10 / 29-34,48 \%$ \\
Doença reumática sistêmica & 5 \\
Granulomatose de Wegener & 2 \\
Artrite reumatóide & 1 \\
Lúpus eritematoso sistêmico & 2 \\
Policondrite recidivante & $6 / 29-20,68 \%$ \\
Doenças infecciosas & 3 \\
Mal de Hansen & 1 \\
Herpes zoster & 2 \\
Herpes simples & $13 / 29-44,82 \%$ \\
Idiopáticas & $21 / 29-72,41 \%$ \\
Tratamento & $12 / 29-41,37 \%$ \\
Corticóide oral & $8 / 29-27,58 \%$ \\
Imunossupressores & \\
Outros & \\
\hline
\end{tabular}




\begin{tabular}{|c|c|c|c|}
\hline & Idiopática $n=13$ & Reumática $\mathrm{n}=10$ & $\mathbf{P}$ \\
\hline Idade média & $51,38 \pm 15,93$ & $48,50 \pm 11,31$ & 0,640 Mann Whitney \\
\hline Sexo & $3 \mathrm{H}: 10 \mathrm{M}$ & $2 \mathrm{H}: 8 \mathrm{M}$ & 1,000 Fisher \\
\hline Forma necrosante & $1 / 13(7,6 \%)$ & $2 / 10(20 \%)$ & 0,550 Fisher \\
\hline Uso de corticóide oral & $8 / 13(61,53 \%)$ & $10 / 10(100 \%)$ & 0,045 Fisher $\left(^{*}\right)$ \\
\hline Uso de imunossupressores & $3 / 13(23,07 \%)$ & $9 / 10(90 \%)$ & 0,002 Fisher $\left(^{*}\right)$ \\
\hline
\end{tabular}

máticas que fortemente influem nas doenças infecciosas. Observa-se na presente série, a ocorrência de 3 casos de Mal de Hansen causando esclerite, doença essa de alta prevalência em nossa região. Um destes pacientes apresentava também infecção pelo vírus da SIDA.

Das doenças reumáticas, a artrite reumatóide é considerada a mais comum neste contexto ${ }^{(4)}$. Todavia, como já comentado anteriormente, este é um panorama em mudança dada às novas possibilidades de tratamento dessa doença trazidas pelo uso de imunobiológicos. Em nossa amostra, a granulomatose de Wegener foi a mais representativa, sendo a esclerite a manifestação inicial da doença em 3 dos 5 casos. Em uma série de 47 pacientes com granulomatose de Wegener descritos por Harper et al. ${ }^{(7)}$, observou-se que 3 pacientes $(6,3 \%$ da amostra estudada) também se apresentaram com manifestações oculares antes das sistêmicas. Esses autores ressaltaram a importância dessa forma de reconhecimento precoce que possibilita o acompanhamento cuidadoso de uma doença com manifestações sistêmicas graves e potencialmente fatais.

Esclerites idiopáticas podem ser consideradas como doenças autoimune órgão-especificas. Apesar do papel da autoimunidade na sua gênese, os mecanismos intrínsecos de sua causa parecem diferir dos casos associados à doença reumática. Um estudo realizado, comparando aspectos anatomopatológicos de olhos enucleados de pacientes com esclerite necrosante de origem reumática com aqueles com a forma idiopática da doença, mostrou que no primeiro caso existia vasculite sugerindo um processo mediado por complexos imunes. Já no caso das formas idiopáticas, os achados anatomopatológicos de reação proliferativa do tecido conjuntivo sem vasculite, eram sugestivos de reação de hipersensibilidade retardada ${ }^{(8)}$. Alguns autores ${ }^{(9)}$, também estudando o aspecto anatomopatológico de pacientes com esclerite, descobriram que, embora nas duas situações houvesse infiltrados ricos em células positivas para CD68 (macrófagos), naquelas com doença reumática subjacente existiam mais células CD20 (linfócitos B).

Do ponto de vista clínico não foi possível encontrar diferenças entre as esclerites idiopáticas e as de origem reumática, exceto pelas formas de tratamento exigidas. Formas mais agressivas de tratamento, com corticóides e imunossupressores, foram necessárias nessas últimas. Todavia esta demanda pode ser parcialmente explicada pelo caráter sistêmico destas doenças das quais o olho reflete a atividade da mesma de maneira bastante fiel.
Enfermidades reumáticas são reconhecidamente associadas com as formas necrosantes da esclerite ${ }^{(3)}$. No presente estudo, apesar de um porcentual mais alto desta forma nos pacientes reumáticos não foi possível demonstrar significância estatística, provavelmente pelo baixo número de componentes da amostra induzindo erro estatístico tipo 2. Para uma análise com poder de $90 \%$, adotando-se $\alpha$ de 0,05 seriam necessários 116 indivíduos em cada grupo. Também não se encontrou diferenças entre a idade dos pacientes com formas reumáticas e idiopáticas. A literatura mostra que uma faixa etária mais avançada está mais comumente associada com esclerites secundárias a doenças sistêmicas ${ }^{(10)}$. Novamente o tamanho da amostra estudada pode ser evocado como causa possível para as diferenças encontradas.

\section{CONCLUSÃO}

Concluindo, pode-se dizer que em nosso meio quase um terço das esclerites tem etiologia reumática e que, apesar destas possuírem uma maior porcentagem de casos necrosantes, não foi possível estabelecer diferenças clínicas entre essas e as idiopáticas.

A cooperação mútua entre oftalmologista e reumatologista se mostrou frutífera no auxílio do esclarecimento etiológico e no manejo desses pacientes com imunossupressores.

\section{ABSTRACT}

Scleritis are entities that may have rheumatic diseases, infections or tumors as etiologic factors or may be a disease restricted only to the eye. Purpose: To share a three year experience of a rheumato-ophthalmologic ambulatory in the diagnosis and treatment of patients with scleritis. Methods: This was a study of 29 cases. All of them were analyzed by the Ophthalmology and Rheumatology Services. Results: From the 29 cases, an underlying disease was found in $55.17 \%$. Rheumatic diseases were present in $34.4 \%$; infectious in $20.6 \%$. Idiophatic forms of scleritis did not differ from the rheumatic ones although a higher number of necrotizing cases were seen in the later group (with no statistical significance). Scleritis due to rheumatic diseases was treated more frequently with oral corticosteroids and immunosuppressive drugs. Con- 
clusions: Patients with scleritis have a high rate of infectious and rheumatic diseases. Cooperation of rheumatologists and ophthalmologists was useful for the etiologic identification of patients with scleritis.

Keywords: Scleritis/etiology; Scleritis/drug therapy; Arthritis, rheumatoid/drug therapy; Wegener granulomatosis/drug therapy; Immunosuppressive agents/therapeutic use

\section{REFERÊNCIAS}

1. Akpek EK, Thorne JE, Qazu FA, Do DV, Jabs DA. Evaluation of patients with scleritis for systemic disease. Ophtalmology. 2004;111(3):501-6. Comment in: Ophthalmology. 2007;114(6):1232.

2. Machado DO, Curi ALL, Fernandes RS, Bessa TF, Campos WR, Oréfice F. Esclerite: características clínicas, associação sistêmica e evolução de 100 pacientes. Arq Bras Oftalmol. 2009;72(2):231-5.
3. Nizam S, Johnstone A, Green M, Gough A. Necrotising scleritis and connective tissue disease: three cases and a review. Clin Rheumatol. 2009;28(3): 339-41.

4. Smith JR, Mackensen F, Rosenbaum JT. Therapy insight: scleritis and its relationship to systemic autoimmune disease. Nat Clin Pract Rheumatol. 2007; 3(4):219-26

5. Jabs DA, Mudun A, Dunn JP, Marsh MJ. Episcleritis and scleritis: clinical features and treatment results. Am J Ophtalmol. 2000;130(4):469-76.

6. Gregersen PK, Plenge MR Gulko PS. Genetics of rheumatoid arthritis. In: Firestein GS, Panayu GS, Wolheim FA, editors. Rheumatoid arthritis. $2^{\text {nd }}$ ed. Oxford: Oxford University Press; 2006. p.3-14.

7. Harper SL, Letko E, Samson CM, Zafirakis P, Sangwan V, Nguyen Q, et al. Wegener's granulomatosis: the relationship between ocular and systemic disease. J Rheumatol. 2001;28(5):1025-32.

8. Riono WP, Hidayat AA, Rao NA. Scleritis: a clinicopathologic study of 55 cases. Ophtalmology. 1999;106(7):1328-33.

9. Usui Y, Parikh J, Goto H, Rao NA. Immunopathology of necrotising scleritis. Br J Ophtalmol. 2008;92(3):417-9.

10. Sainz De La Maza SM, Escobar Barranco JL, Foster CS. [Characteristics of scleritis in patients older than 60 years]. Arch Soc Esp Oftalmol. 2001;76(7): 425-30. Spanish.

\title{
III \\ 58 a Jornada Científica do Centro Brasileiro de Estrabismo
}
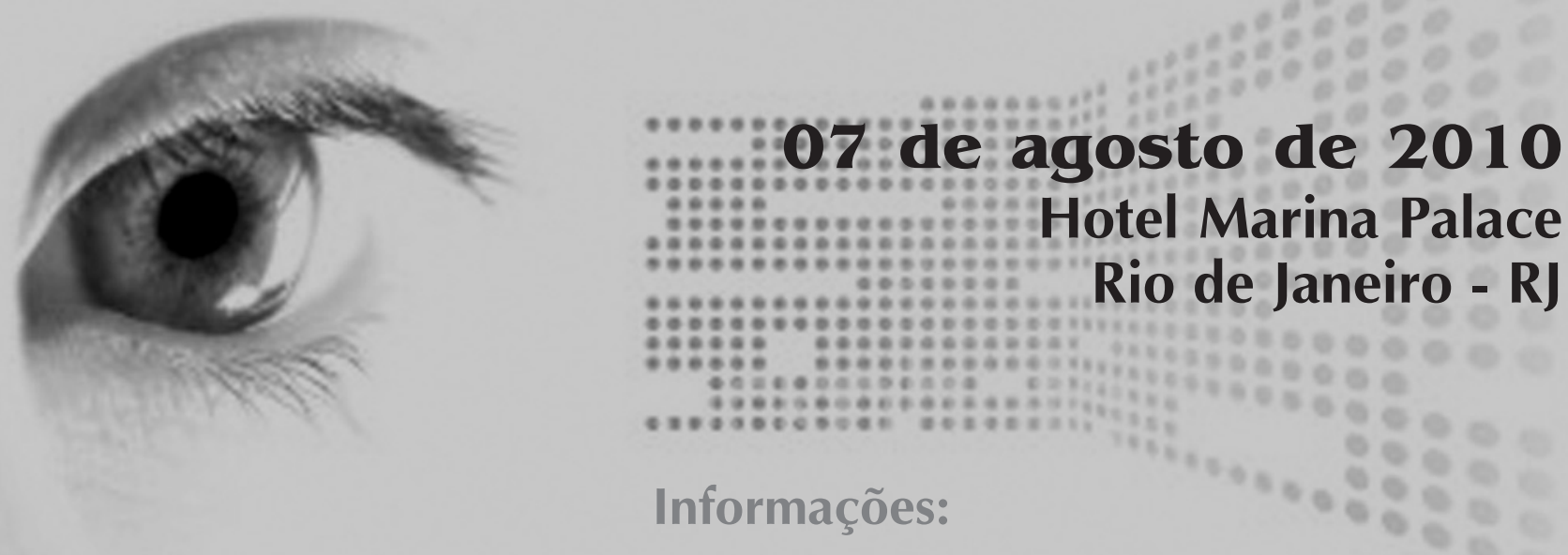

\author{
Tel.: (21) 3547-1680 \\ e-mail: cbe@cbo.com.br \\ site: www.nobilistur.com.br
}

